\title{
Amaury CHAUOU, L'idéologie Plantagenêt. Royauté arthurienne et monarchie politique dans l'espace Plantagenêt (XIIe-XIIIe siècles)
}

Lionel Rousselot

\section{(2) OpenEdition \\ 1 Journals}

\section{Édition électronique}

URL : https://journals.openedition.org/abpo/1640

DOI : 10.4000/abpo.1640

ISBN : 978-2-7535-1485-0

ISSN : $2108-6443$

\section{Éditeur}

Presses universitaires de Rennes

\section{Édition imprimée}

Date de publication : 20 mars 2002

Pagination : 166-168

ISBN : 978-2-86847-708-8

ISSN : 0399-0826

\section{Référence électronique}

Lionel Rousselot, « Amaury CHAUOU, L'idéologie Plantagenêt. Royauté arthurienne et monarchie politique dans l'espace Plantagenêt (XIle-XIIle siècles) », Annales de Bretagne et des Pays de l'Ouest [En ligne],

109-1 | 2002, mis en ligne le 20 mars 2004, consulté le 22 juillet 2022. URL : http://

journals.openedition.org/abpo/1640; DOI : https://doi.org/10.4000/abpo.1640 
res contrôlés par les moines et dans les régaires ecclésiastiques, et réfuterait également le rôle de la motte en matière de défrichement.

L'imposante annexe 2, qui occupe un dixième de l'ouvrage, offre un inventaire des mottes par communes avec une mise en évidence des lignages qui les détiennent. La connaissance de ces derniers est résumée dans quelques 34 tableaux généalogiques qui viennent s'ajouter à ceux figurant dans le texte. Cet inventaire complète celui publié en 1990, par le même auteur (Les mottes médiévales d'Ille-et-Vilaine, Rennes, 1990). Il est regrettable que les impératifs d'édition aient obligé à la suppression des sources de cette annexe et de près des deux tiers des relevés de sites figurant dans la thèse. Un renvoi aux relevés inclus dans le corps du texte eût été pratique.

Au total, M. Brand'Honneur nous offre, sur la base d'une étude rigoureuse des sources et des lieux une vision renouvelée du château, du manoir et de la société chevaleresque aux $\mathrm{XI}^{\mathrm{e}}$ et $\mathrm{XII}^{\mathrm{e}}$ siècles. S'inscrivant parfois en contradiction, souvent volontairement un peu en recul par rapport aux travaux ayant contribué à la connaissance du phénomène au cours de ces vingt dernières années, ce travail suscitera sans doute des commentaires, preuve qu'il aura alors rempli sa vocation première.

Christophe АмІот

Chauou, Amaury, L'idéologie Plantagenêt. Royauté arthurienne et monarchie politique dans l'espace Plantagenêt (XII - -XIII ${ }^{e}$ siècles), Rennes, Presses Universitaires de Rennes, collection « Histoire ", 2001, 324 pages.

Amaury Chauou offre ici, à destination d'un large public, la version remaniée et préfacée par Hervé Martin de la thèse qu'il a soutenue à l'université de Rennes 2 Haute Bretagne en janvier 2000. Dans cette étude dense, l'auteur s'est employé à cerner et à analyser la naissance et la diffusion aux XII et XIII' siècles de "L'idéologie Plantagenêt ", soit " les thèmes et les schémas idéologiques en rapport avec la fonction monarchique, qui ont pu servir la construction de l'État Plantagenêt " (p. 24). Face à une idéologie royale capétienne en plein essor et dans un contexte d'affirmation des monarchies féodales, Henri II Plantagenêt, promu roi d'Angleterre dans un pays à peine sorti des affres de la guerre civile et à la tête d'un ensemble de possessions continentales, a en effet rapidement ressenti la nécessité de forger une idéologie cohérente qui lui permettrait de légitimer son pouvoir et de souder autour de sa personne l'aristocratie sur la totalité de ses domaines. Pour répondre à cet enjeu de taille, la figure d'Arthur, chef breton du $\mathrm{Vl}^{\mathrm{e}}$ siècle devenu personnage légendaire en particulier sous la plume de Geoffroy de Monmouth, a été mobilisée et l'imaginaire qu'il véhiculait récupéré afin de servir les intérêts politiques de la dynastie. À la suite des travaux de Jacques Krynen ou de Colette Beaune pour la France, A. Chauou s'est donc attaché à une étude des idées et des représentations politiques au sein du vaste espace Plantagenêt.

Pour ce faire, outre une bibliographie abondante (plus de 600 titres tant en français qu'en langues étrangères!), l'auteur a dépouillé la production littéraire de la cour Plantagenêt, foyer culturel de toute première importance dans l'Occident des XII ${ }^{\mathrm{e}}$-XIII ${ }^{\mathrm{e}}$ siècles. Lourde tâche tant les sources sont nombreuses et variées : chroniques célébrant les ancêtres de la dynastie ou Henri II lui-même, écrits politiques de Jean de Salisbury et d'Étienne de Fougères, poèmes histo- 
riques de Wace et de Benoît de Sainte-Maure, romans antiques, lais de Marie de France et romans de Chrétien de Troyes... [chapitres I, II, III]. Bien sûr, toutes ces œuvres n'ont pas directement été commandées par les souverains angevins et il serait même absurde d'assimiler leur cour à " un atelier de propagande organisée à grande échelle, rassemblant des exécutants recrutés et appointés ad hoc " (p. 124). Les œuvres de commande sont même fort peu nombreuses, ce qui, contre certaines tendances de l'historiographie actuelle, ne permet pas malgré tout de dénier tout rôle culturel à Henri II Plantagenêt et Aliénor d'Aquitaine, comme le montre de façon convaincante A. Chauou (p. 80-87). En fait, le prestige du couple royal et le rayonnement de la cour Plantagenêt, au carrefour de traditions insulaires et continentales, ont été tels que nombre d'auteurs ont alors servi, de façon plus ou moins consciente, les entreprises de la dynastie angevine en puisant dans la matière de Bretagne et en faisant circuler des thèmes et des représentations politiques de la société féodale qui pouvaient lui être favorables. Cette " propagande diffuse ", concept que l'auteur emprunte à Jacques Le Goff car parler de manipulation idéologique n'a guère de sens dans le contexte des sociétés des $\mathrm{XII}^{\mathrm{e}}$-XIII ${ }^{\mathrm{e}}$ siècles, a paré Henri II et son gouvernement des valeurs du monde arthurien.

La royauté Plantagenêt, véritable " carrefour idéologique » [chapitre IV], s'est ainsi trouvée à la confluence de deux visions de la fonction monarchique. L'une, d'inspiration féodale et laïque, a présenté Arthur sous les traits du Primus inter pares et privilégié l'image du roi combattant, de telle sorte qu'Henri II Plantagenêt a pu se prévaloir d'être le digne successeur de celui qui était dépeint comme le modèle du roi-chevalier. L'autre, issue du courant augustiniste et grégorien, a insisté sur la majesté d'Arthur et la souveraineté de l'office royal. La Table Ronde, au cœur de l'imaginaire arthurien, illustre cette double réflexion politique selon qu'Arthur y est représenté siégeant dans une position similaire à celle de ses chevaliers ou qu'au contraire il apparaît en retrait, assis à une table d'honneur, afin de manifester sa prééminence sur l'aristocratie. Loin d'être contradictoires pour Henri II, ces deux visions de l'exercice de l'autorité royale ont au contraire pleinement servi les intérêts politiques de celui qui se devait de concilier, en tant que roi d'Angleterre et vassal du roi de France pour ses possessions continentales, les notions de féodalité et de souveraineté. Appuyée sur les thèmes de la translatio studii et de la translatio imperii que les auteurs proches de la cour angevine n'ont pas manqué de développer [chapitre V], l'idéologie Plantagenêt s'est donc efforcée d'apparaître comme un ensemble cohérent pour le plus grand profit de la dynastie régnante.

L'élaboration de cette idéologie a atteint son point d'orgue sous le règne de Richard Cœur-de-Lion lorsqu'ont été mises au jour en 1191 les tombes supposées d'Arthur et de Guenièvre à l'abbaye de Glastonbury [chapitre VI]. Vaste supercherie destinée à restaurer le prestige d'un établissement monastique en déclin ou véritable découverte de sépultures attribuées à ce couple mythique? A. Chauou fait le point sur la question et montre surtout que ce " télescopage du mythe et de la réalité " (p. 204) assoit définitivement le contrôle des Plantagenêts sur la légende arthurienne. À une époque où les peuples d'origine brittonique croyaient en un retour d'Arthur, leur ancien roi, l'invention de Glastonbury a en effet sonné le glas de leurs espérances et anéanti le messianisme arthurien, particulièrement vif en cette fin du XII ${ }^{\mathrm{e}}$ siècle, notamment chez les Bretons d'Armorique, et politiquement dangereux pour les Plantagenêts. On peut d'ailleurs regretter que, dans ce chapitre passionnant, l'auteur n'ait pas davantage montré combien la dynastie angevine, dans son entreprise de récupération de la figure d'Arthur, est entrée en concurrence avec les tradi- 
tions culturelles et les aspirations des peuples celtiques qui étaient sous sa domination ${ }^{1}$.

Dès lors, fondée sur un ensemble d'œuvres au rayonnement toutefois inégal [chapitre VII] et enracinée dans une abbaye identifiée au lieu mythique d'Avalon, cette idéologie a survécu à son fondateur ainsi qu'à la disparition de l'empire Plantagenêt et a continué à servir à des degrés divers les intérêts politiques des rois du XIII ${ }^{\mathrm{e}}$ siècle [chapitre VIII]. De tous, c'est sans conteste Édouard Ir qui a le plus tiré parti des symboles du monde arthurien au point d'assimiler sa cour à celle du roi Arthur : transfert de la couronne d'Arthur à Westminster, réorganisation de la nécropole de Glastonbury, organisation de Tables Rondes... La matière de Bretagne a alors été mobilisée pour justifier les conquêtes du Pays de Galles et de l'Écosse et renforcer les liens entre Édouard Ir et les couches les plus modestes de son aristocratie. En ce début du XIV ${ }^{\mathrm{e}}$ siècle, l'exploitation de la figure arthurienne est encore loin d'être achevée et, comme le souligne A. Chauou, Édouard III a aussi été un grand orchestrateur à des fins politiques de la geste arthurienne. Née dans le cadre du vaste espace Plantagenêt, l'idéologie forgée au temps de Henri II a donc participé jusqu'à la fin du Moyen Âge à la construction de l'État royal anglais.

Outre la rigueur de la construction, la richesse du propos et la clarté de l'argumentation, le lecteur appréciera tout particulièrement dans ce travail les larges extraits de documents cités, en particulier ceux se rapportant à l'exhumation d'Arthur et de Guenièvre, ainsi que les va-et-vient constants que l'auteur opère entre un corpus d'interprétation parfois délicate et le contexte politique des $\mathrm{XII}^{\mathrm{e}}$-XIII ${ }^{\mathrm{e}}$ siècles. On l'aura compris, en explorant l'utilisation que les Plantagenêts ont faite de la matière de Bretagne, A. Chauou apporte une contribution importante à l'étude des représentations politiques au Moyen Âge. Nul doute que cet ouvrage, à la charnière de l'histoire politique et de l'histoire culturelle, rencontrera un large écho auprès de tous les passionnés d'histoire du Moyen Âge et de tous ceux que la littérature arthurienne fascine.

Lionel ROUSSELOT

JANKULAK, Karen, The Medieval cult of st Petrock, Studies in Celtic History XIX, The Boydell Press, Woodbridge, 2000.

Durant le Moyen Âge, saint Pétroc est le plus célèbre des saints de Cornwall où son culte est largement diffusé au moins dès le $\mathrm{x}^{\mathrm{e}}$ siècle. Celui-ci est attesté en Basse Bretagne à la même époque et il se diffuse en Haute Bretagne au XII ${ }^{\mathrm{e}}$ siècle. La détention des reliques de son saint patron conférait au prieuré de Saint-Petroc de Bodmin en Cornwall une assise foncière et une influence hors du commun dans la région. C'est sans doute pourquoi les chanoines de Bodmin ont doté saint Pétroc d'un riche dossier hagiographique qui se distingue par sa diversité : deux Vitae en prose, une Vita metrica, un recueil de Miracula et un récit de translation (De reliquiarum furto). En effet, en 1177, les reliques du saint furent dérobées dans sa fondation de Bodmin et emportée à l'abbaye de Saint-Méen, en Bretagne. Elles furent par la suite restituées, en partie grâce à l'intervention d'Henri II Plantagenêt.

1. Cette question a été abordée par Jean-Christophe Cassard, " Arthur est vivant! Jalons pour une enquête sur le messianisme royal au Moyen Âge ", Cahiers de civilisation médiévale, XXXII, avril-juin 1989, p. 135-146. 